\title{
Posterior cruciate ligament injury is influenced by intercondylar shape and tibial eminence size.
}

\section{Abstract}

Background: Little is known about the risk factors for sustaining a PCL rupture. Finding risk factors is the first step to trying to prevent PCL ruptures from occurring.

Hypothesis: The shape of the knee of patients who obtain a PCL rupture is different from the shape of the knee of control patients.

Study design: Case-control study; Level of evidence, 3.

Methods: We compared the Anterior-Posterior view X-rays, lateral view X-rays and Rosenberg view X-rays of 94 patients with a ruptured PCL to a control group of 168 patients matched for gender with an intact PCL after knee trauma. Statistical shape modelling software was used to assess knee shape and find differences in anatomical landmarks between both groups.

Results: Both on the Anterior-Posterior view X-rays as on the Rosenberg view X-rays, we found shape variants to be significantly different between patients who tore their PCL compared to patients with an intact PCL after knee trauma. Overall, patients who ruptured their PCL have smaller intercondylar notches and a smaller tibial eminence than control patients. 
Conclusions: Patients with a PCL rupture have a smaller intercondylar notch and a smaller tibial eminence compared to patients with an intact $\mathrm{PCL}$ rupture after knee trauma.

Keywords: PCL; femoral intercondylar notch; knee anatomy; PCL prevention; PCL risk factors

\section{What is known about this subject}

Posterior cruciate ligament injury is far less investigated than anterior cruciate ligament injury. Perhaps because the incidence is less great than the incidence of $A C L$ injury, but with more people participating in sporting activities, these injuries will potentially increase in the future. Till date, there are no risk factors identified with regard to sustaining a PCL rupture. There have been numerous of studies published about risk factors for sustaining an ACL rupture. When risk factors for a PCL rupture can be identified, research can start focusing on searching for ways to prevent a PCL rupture. For example with specified training programs in athletes. These programs have proven to work for patients at risk for an $\mathrm{ACL}$ rupture.

\section{What this study adds to existing knowledge}

To our knowledge, our study is the first one to provide evidence that there are anatomical differences between patients with a PCL rupture and control patients. Off course, more research is needed to confirm our findings, preferable in large prospectavily followed groups. But the fact that the findings of our current study are consistent with findings in $A C L$ injured knees suggests that the morphology of the intercondylar notch plays an important role in both $\mathrm{PCL}$ and $\mathrm{ACL}$ ruptures. Our results could be used in upcoming research into the risk factors for PCL ruptures and perhaps, in the near future, help to identify individual 
patients who are at greater risk for sustaining a PCL rupture. Finding risk factors is essential if we ever want to be able to prevent PCL ruptures from happening.

\section{Introduction}

Identifying risk factors for sustaining a PCL rupture could be useful in preventing a PCL rupture (1).

Till date, there are no risk factors identified with regard to sustaining a PCL rupture. The role of osseous anatomical variation in a traumatic knee has been investigated in ACL-deficient knees (2). Statistical Shape modeling is a hypothesis generating model, which can be used to predict which patients have an increased risk of a rupture or have good or worse clinical outcome in relation with the shape of the knee(3).

The posterior cruciate ligament $(P C L)$ is the strongest ligament of the knee $(4,5)$. The exact incidence rate of a PCL rupture remains uncertain. Based on clinical setting in the emergency department., incidence reports of a PCL rupture vary from $4 \%(6)$ till as high as $38 \%(7)$ of all traumatic knee injuries, seen there. With more people participating in sporting activities, these injuries will potentially increase in the future. At short-term follow up a PCL rupture causes pain, posterior laxity and reduces sports abilities. Over the long-term, a PCL rupture is a risk factor for developing osteoarthritis (8-11).

When shape variants of the knee are identified, it is helpful for screening patients who are prone to a PCL rupture, for example during medical screening in athletes. Patients with an increased risk could be counseled and offered additional training of the knee to reduce this risk. These training programs are only cost-efficient when patients are selected with a high risk of sustaining a PCL rupture. This has proven efficacy in patients prone for $\mathrm{ACL}$ rupture $(12,13)$.

The shape of the knee can be assessed using standard radiographs, and in general a radiograph will be obtained after a knee injury or at medical screening for athletes. Because of this widespread availability and low cost of radiographs, it would be very useful to identify subjects prone to PCL rupture. 
The purpose of our study is to identify any osseous knee shape variations which are related to a PCL.

\section{Patients and methods}

We performed a case-control study. All patients with a traumatic knee event that visited the outpatient clinic of our hospital (name blinded for review) in the period of January 2003 till May 2014 were eligible.

\section{Cases}

Patients were selected as cases after a traumatic knee event, with a PCL rupture confirmed either by MRI or by arthroscopy.

\section{Controls}

Controls were selected after a knee injury comprising of: 1) meniscus tear; 2) combined medial collateral ligament and meniscus injury. 3) A proven intact PCL and anterior cruciate ligament $(A C L)$, confirmed by MRI or arthroscopy after the occurrence of the knee trauma;

Selection criteria of our patients and controls were the availability of a lateral view $\mathrm{x}$-ray and anterior posterior (AP) view x-ray. We also selected the Rosenberg view x-rays, when available. Controls had to be practicing sports at the moment of the knee trauma. Patients and controls had Kellgren and Lawrence grade $0-1$ at presentation(14). There were no significant differences between possible confounders such as gender, age and BMI.

\section{X-rays and Statistical Shape Modeling.}

The radiological measurements were performed on standard non-weight bearing lateral view radiographs, AP view radiographs and Rosenberg view radiographs. On the radiographs, we outlined 
the distal femur, the proximal tibia and fibula (ASM tool kit, Manchester University, Manchester, UK). The shape of the distal femur and proximal tibia were defined by 60 landmark points for the lateral view and 25 landmark points for AP view and the Rosenberg view which were placed along the surface of the bone on the x-rays. Each point was placed on the same location in each image to allow comparison between shapes (figure).

Principal component analysis was used to transform the set of points into a statistical shape model, which comprises a number of shape variants that together explain $95 \%$ of variation in shape of the knee of the study population. Intra-observer reliability was assessed by outlining $25 \mathrm{x}$-rays a second time two weeks later. These 25 x-rays were blinded for the observer. The description of what shape aspects a variant represents was determined at a consensus meeting.

\section{Statistical analysis}

The association between the shape of the knee and whether or not patients had a ruptured PCL was analyzed by logistic regression analyses. The presence / absence of a PCL rupture was used as dependent variable and the different shape variants as independent variables.

In total 30 shape variations were found per $\mathrm{x}$-ray view. To correct for multiple testing we applied Bonferroni correction for multiple comparisons $(0.05 / 30=0.002)$. All Statistical analyses were performed with IBM SPSS Statistics for Windows (Version 20.0. Armonk, NY: IBM Corp).

\section{Results}

\section{Patients}

Of all the patients seen in the period 2003-2014 with a knee trauma, 94 patients with a PCL rupture were eligible and were included. Of the control patients, 168 patients without a PCL rupture (all with AP, Lateral and Rosenberg view X-rays) met the inclusion criteria (see figure 1). Of 42 patients with a 
PCL rupture Rosenberg view x-ray were available. Patients' characteristics are shown in table 1 . The diagnosis of the individual control patients are shown in table 2.

Table 1

Patient Demographic characteristics

\begin{tabular}{llll}
\hline & $P C L$ injured & Control group & $P-$ \\
& $(n=94)$ & $(n=168)$ & value \\
\hline Age, years & $40 \pm 13.6$ & $38 \pm 12.0$ & .148 \\
BMI, $\mathrm{kg} / \mathrm{m}^{2}$ & $25 \pm 3.0$ & $25 \pm 3.2$ & .873 \\
Sex (female) $-\mathrm{n}(\%)$ & $24(26)$ & $49(29)$ & .940 \\
Time between & & & .002 \\
trauma and X-ray & $12.6 \pm 16$ & $6.9 \pm 10$ & \\
(months) & & & \\
\hline Data are expressed as mean \pm standard deviation & & \\
Body mass index (BMI). &
\end{tabular}


Table 2

Diagnosis of control patients

\begin{tabular}{lcc}
\hline Diagnosis, $\mathrm{n}(\%)$ & & \\
\hline Medial meniscus tear & 57 & $(33.9)$ \\
Lateral meniscus tear & 32 & $(19)$ \\
Cartilage lesion & 15 & $(8.9)$ \\
Bone contusion & 11 & $(6.5)$ \\
Collateral ligament lesion & 7 & $(4.2)$ \\
No intra-articular lesions & 46 & $(27.4)$ \\
\hline
\end{tabular}

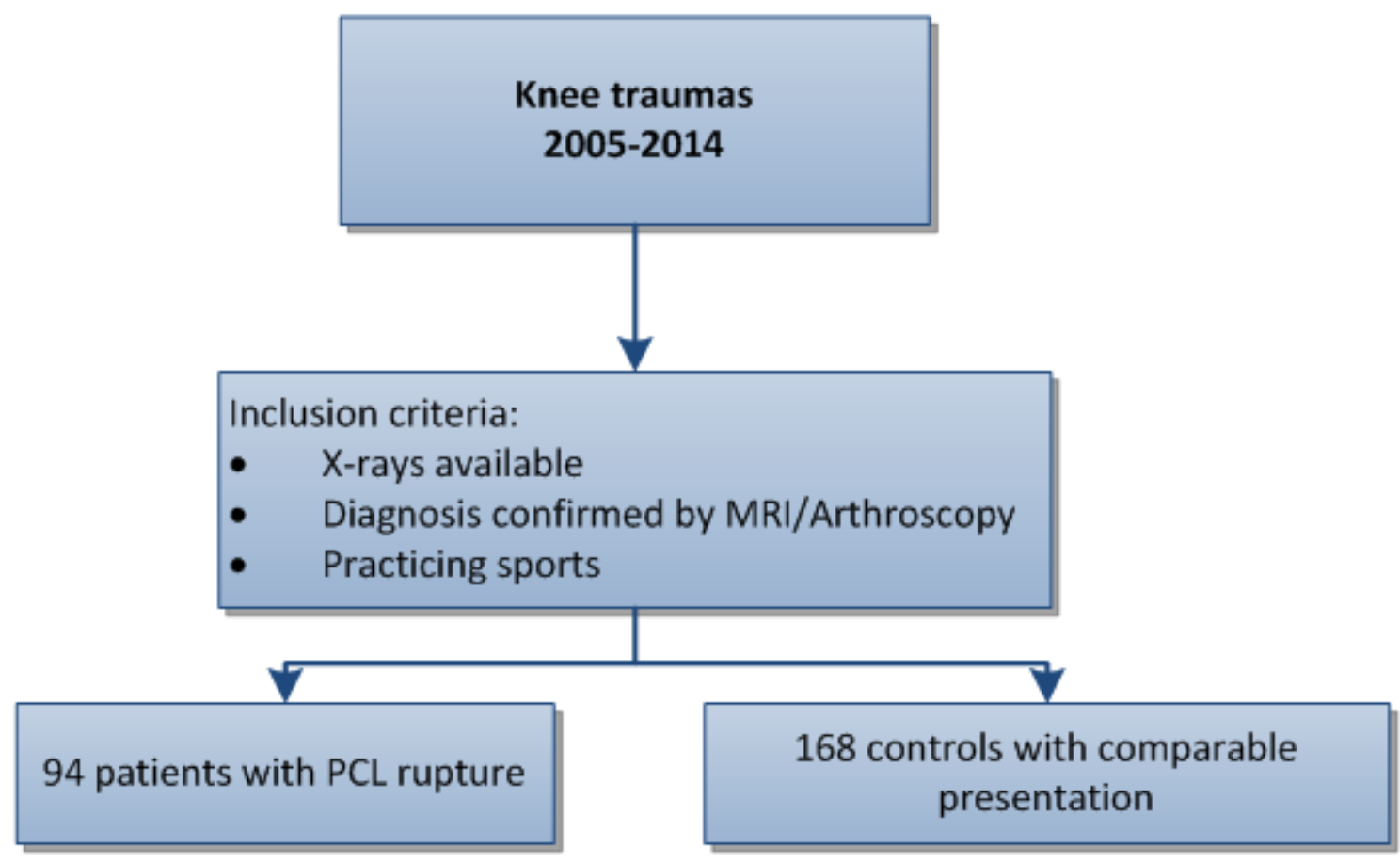

Figure 1: Flowchart of patients included in the study.

\section{Shape variants and $\mathrm{PCL}$ rupture}

Statistical Shape Modelling provided 30 shape variants for the lateral, 30 shape variants for the Rosenberg and 30 shape variants for the AP view $x$-rays (variant 0 to variant 29). The intra-observer ICC of the placement of the points and the thereafter found different modes were considered good, with a mean of 0.81 (0.48 to 0.97 ), with $89 \%$ of the variants having an ICC above 0.7 . 
For the lateral view $\mathrm{x}$-rays we found two shape variants that were significantly different between cases and controls (table 3). However while analyzing these shape variants it became clear they were due to positioning of the knee while the x-ray was taken. For the completeness of the article we decided to publish these results, although they are not caused by variation in shape of the knee itself. For the AP view $\mathrm{x}$-rays we found 2 variants that were significantly different between patients with a PCL rupture and controls; For the Rosenberg view we found 3 variants that were significantly different between patients with a PCL rupture and controls.

\section{Description of the significant shape variants.}

Below we present a description of the variants significantly associated with PCL rupture. The software produces graphics, of which the extremes (+2SD and -2SD) for each variant are depicted in figure 2 and 3 on the left and right side. In the middle an overlay is presented. Higher variants describe more subtle shape aspects, e.g. the variation in shape represented in , for example, variant 17 is a much more subtle than the variation represented by variant 1 . 
Table 3

relation between shape variants and $\mathrm{PCL}$ rupture

$\begin{array}{lll}\text { Odds ratio } & 95 \% \text { C.I. } & \text { P-value }\end{array}$

\section{AP view}

$\begin{array}{llll}\text { Variant } 13 & 1.6 & (1.2-2.2) & .001 \\ \text { Variant } 22 & 1.6 & (1.2-2.1) & .001\end{array}$

Rosenberg view

$\begin{array}{lccc}\text { Variant 1 } & 0.4 & (0.3-0.6) & <0.001 \\ \text { Variant 6 } & 0.5 & (0.3-0.7) & .001 \\ \text { Variant 9 } & 1.9 & (1.3-2.7) & <0.001\end{array}$

Lateral view

$\underline{\text { Position artifact }}$

Variant 0

1.6

$(1.2-2.1)$

.0015

Variant 1

0.001

$(0.001-0.008)$

$<0.001$

\section{Anterior-Posterior view}

The intercondylar notch was significantly different between patients with a PCL rupture and healthy control patients. This was found in both variant 13 and variant 22 on the AP view $\mathrm{x}$-rays, as shown in figure 2. Patients with a PCL rupture had on average a relatively smaller width of the intercondylar notch than patients who had an intact PCL. Variant 22 showed a difference in the shape of the tibial eminence. Patients with a PCL rupture had on average higher tibial eminences than patients who did not have a PCL rupture. 


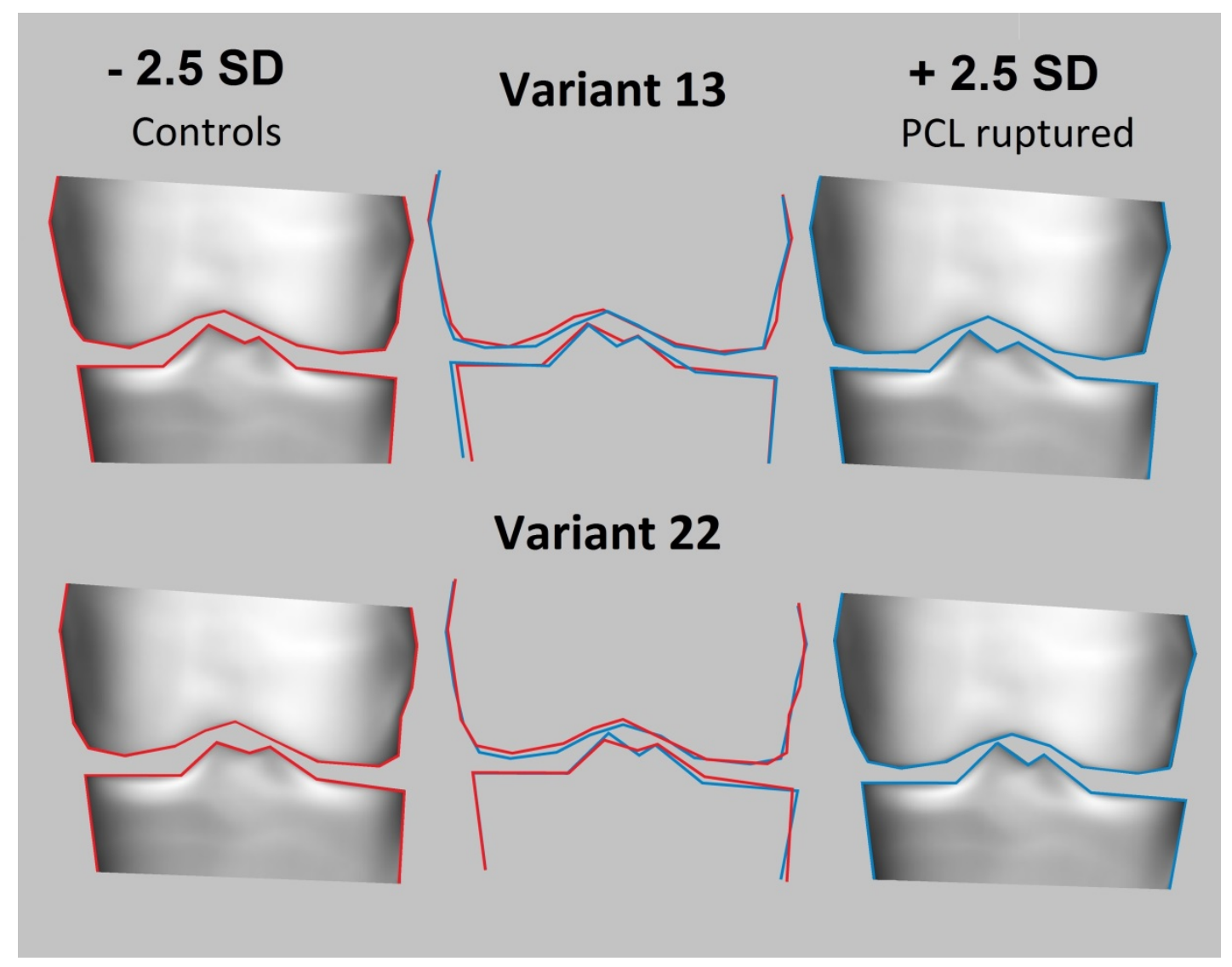

Figure 2: Graphic outcomes of Statistic shape modelling: Two variants that are significantly different for patients with intact and ruptured PCL. On the left and right side are the two extremes (+/- $2.5 \mathrm{SD})$; in the middle the overlay of both sides. SD = Standard Deviation

\section{$\underline{\text { Rosenberg view }}$}

The intercondylar notch was significantly different between patients with a PCL rupture and healthy control patients. This was found in variant 1, variant 6 and variant 9 on the Rosenberg view x-rays, as shown in figure 3. Patients with a PCL rupture had on average narrower intercondylar notches than patients with an intact PCL. Variant 6 showed a difference in the shape of the tibial eminence.

Patients with a PCL rupture had on average higher tibial eminences than patients who did not have a PCL rupture. 


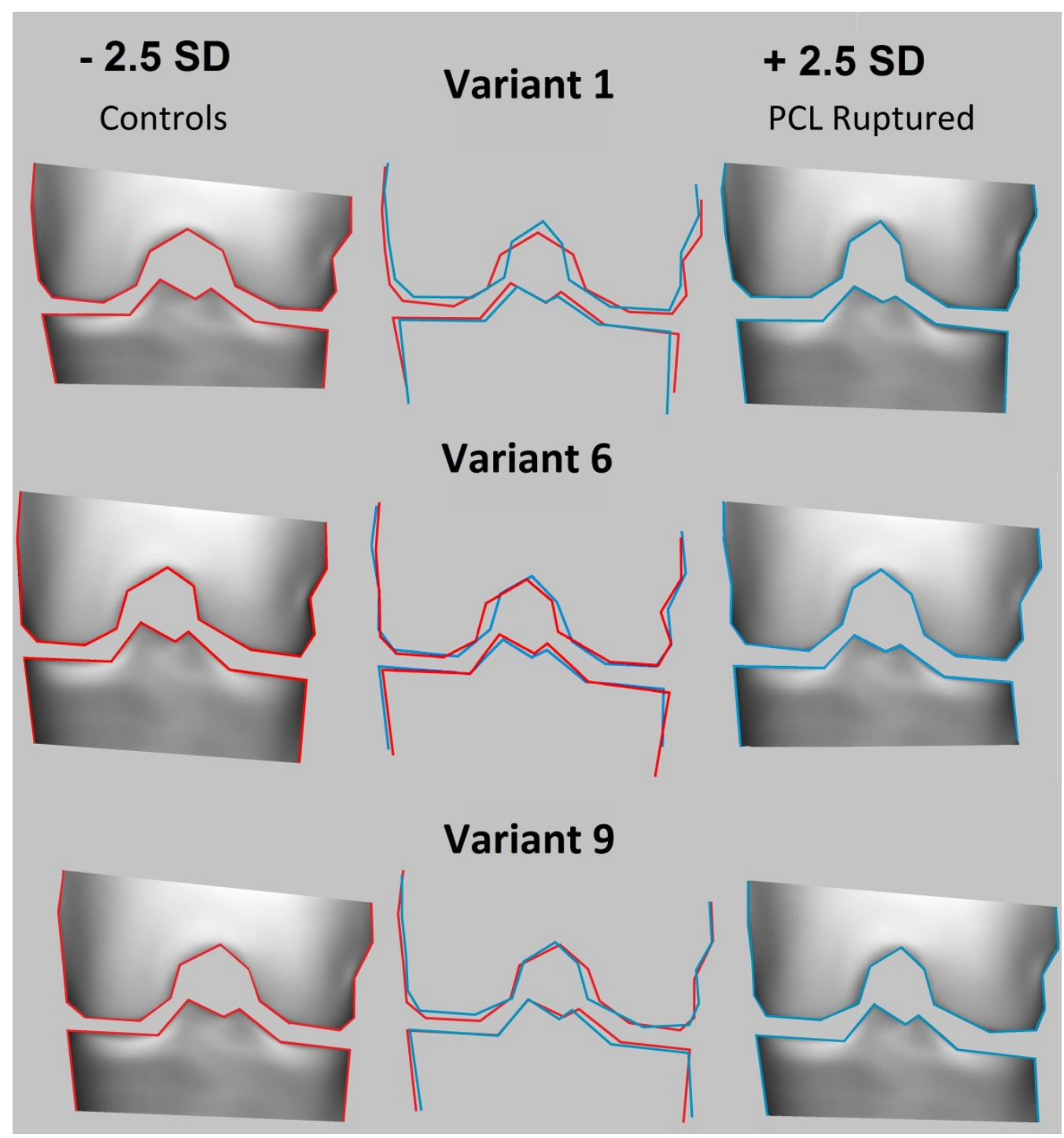

Figure 3: Graphic outcomes of Statistic shape modelling: five variants that are significantly different for patients with intact and ruptured PCL. On the left and right side are the two extremes (+/- 2.5 SD); in the middle the overlay of both sides. SD = Standard Deviation

\section{$\underline{\text { Lateral view }}$}

Variant 1 showed a variation in flexion and extension in the knee. Patients with a PCL injury had on average more flexion in the knee than patients who did not have a PCL injury.

Variant 2 is a variation in rotation of the tibia. Patients with -SD in variant 2 had a Blumensaat line that crossed at a steep angle with the midline of the femur. Patients with +SD seemed to have a larger angle with the midline of the femur. We assumed that this could be explained by a variation in knee flexion during the taking of the X-ray. Also, patients with -SD seemed to have a less wide tibia in 
comparison to patients who scored $+S D$ in variant 2. Patients with a PCL injury scored higher (+SD) on variant 2 than patients who did not have a PCL injury (-SD).

\section{Discussion}

The main finding of the present study is that there are significant differences in bony morphology on the AP view and Rosenberg view $x$-ray between patients with a ruptured PCL and control patients. A smaller and more sharply angled intercondylar notch and a more wider tibial eminence are related to a PCL rupture.

Previously conducted research showed that patients with a smaller intercondylar notch also have a smaller PCL and a smaller ACL $(15,16)$. A smaller PCL can endure less force than a larger PCL. So combined with our present study it is possible that a patient with a smaller intercondylar notch, also has a smaller PCL resulting in an increased rupture risk. An alternative explanation is that a smaller intercondylar notch gives impingement of the PCL by surrounding structures. Triantafyllidi et al (17), showed that the PCL occupies most of the space of the intercondylar notch in flexion, possibly resulting in impingement of the $\mathrm{PCL}$ in this position.

The findings on the lateral view are explained by variation in positioning of the knee when the $x$-ray is taken. The difference found is thought to be the result of difference in flexion and extension positioning during the taking of the knee X-ray. When we examined the different variants in our consensus meeting, we viewed 3D, moving animations of the shape variants, on this animation the differences are more clearly visible than on 2D images.

Research into risk factors for sustaining a PCL rupture is lacking. To our knowledge, our study is the first one to provide evidence that there are anatomical differences between patients with a PCL rupture and control patients. Our results could be used in upcoming research into the risk factors for PCL ruptures and perhaps, in the near future, help to identify individual patients who are at greater 
risk for sustaining a PCL rupture. Finding risk factors is essential if we ever want to be able to prevent PCL ruptures from happening.

Numerous studies into the bony morphology of the knee have focused on patients with an $A C L$ rupture (18-33). Recently research had been published that found significant differences in the shape of the knee between patients with an ACL rupture and control patients. The findings of this study were consistent with the findings in our present study. The morphology of the intercondylar notch plays an important role in both $\mathrm{PCL}$ and $\mathrm{ACL}$ ruptures.

A drawback of Statistical Shape Modelling is that it is not immediately clear what a shape variant specifically represents as is the case with predefined morphological measures. We used an expert consensus meeting to determine which shape aspects were captured in each variant.

We specifically included patients after a knee trauma and compared PCL to none PCL trauma patients. This makes the two selected groups of patients more comparable than when we would have selected healthy controls without a traumatic event. Radiographs were used for assessing the shape of the knee. The benefits of the use of radiographs are that they are widely available, relatively inexpensive. At our hospital patients with a traumatic knee have as an entrance work-up, $x$-rays of the knee taken, thus the results of this study have a higher reproducibility and increased generalizability.

An advantage of Statistical Shape Modelling is that the various variants represent relative variation in shape, independent of differences in size of the joint. In this way the method reduces errors caused by variation in magnification or in size variation of the patient's knee.

Conclusion

This study demonstrates that differences in the shape of the knee are related to the presence of a PCL rupture after a traumatic event of the knee. A smaller and more sharply angled intercondylar 
notch and a more flattened tibial eminence are related to a PCL rupture. This suggests that the shape of the knee is a risk factor for sustaining a PCL rupture. Further research should focus on these points of interest to find predictors of PCL rupture.

1. McAllister DR, Petrigliano FA. Diagnosis and treatment of posterior cruciate ligament injuries. Current sports medicine reports. 2007;6(5):293-9. Epub 2007/09/22.

2. Eggerding V, van Kuijk KS, van Meer BL, Bierma-Zeinstra SM, van Arkel ER, Reijman M, et al. Knee shape might predict clinical outcome after an anterior cruciate ligament rupture. The bone \& joint journal. 2014;96-B(6):737-42. Epub 2014/06/04.

3. Cootes TF, Taylor CJ, Cooper DH, Graham J. Active Shape Models-Their Training and Application. Computer Vision and Image Understanding. 1995;61(1):38-59.

4. Kennedy JC, Hawkins RJ, Willis RB, Danylchuck KD. Tension studies of human knee ligaments. Yield point, ultimate failure, and disruption of the cruciate and tibial collateral ligaments. The Journal of bone and joint surgery American volume. 1976;58(3):350-5. Epub 1976/04/01.

5. Trent PS, Walker PS, Wolf B. Ligament length patterns, strength, and rotational axes of the knee joint. Clinical orthopaedics and related research. 1976(117):263-70. Epub 1976/06/01.

6. Bollen S. Epidemiology of knee injuries: diagnosis and triage. British journal of sports medicine. 2000;34(3):227-8. Epub 2000/06/15.

7. Fanelli GC, Edson CJ. Posterior cruciate ligament injuries in trauma patients: Part II. Arthroscopy : the journal of arthroscopic \& related surgery : official publication of the Arthroscopy Association of North America and the International Arthroscopy Association. 1995;11(5):526-9. Epub 1995/10/01.

8. Logan M, Williams A, Lavelle J, Gedroyc W, Freeman M. The effect of posterior cruciate ligament deficiency on knee kinematics. The American journal of sports medicine. 2004;32(8):191522. Epub 2004/12/02.

9. MacDonald P, Miniaci A, Fowler P, Marks P, Finlay B. A biomechanical analysis of joint contact forces in the posterior cruciate deficient knee. Knee surgery, sports traumatology, arthroscopy : official journal of the ESSKA. 1996;3(4):252-5. Epub 1996/01/01.

10. Shelbourne KD, Clark M, Gray T. Minimum 10-year follow-up of patients after an acute, isolated posterior cruciate ligament injury treated nonoperatively. The American journal of sports medicine. 2013;41(7):1526-33. Epub 2013/05/09.

11. Lee BK, Nam SW. Rupture of posterior cruciate ligament: diagnosis and treatment principles. Knee surgery \& related research. 2011;23(3):135-41. Epub 2012/05/10.

12. Swart E, Redler L, Fabricant PD, Mandelbaum BR, Ahmad CS, Wang YC. Prevention and screening programs for anterior cruciate ligament injuries in young athletes: a cost-effectiveness analysis. The Journal of bone and joint surgery American volume. 2014;96(9):705-11. Epub 2014/05/09.

13. Donnell-Fink LA, Klara K, Collins JE, Yang HY, Goczalk MG, Katz JN, et al. Effectiveness of Knee Injury and Anterior Cruciate Ligament Tear Prevention Programs: A Meta-Analysis. PloS one. 2015;10(12):e0144063. Epub 2015/12/05.

14. Kellgren JH, Lawrence JS. Radiological assessment of osteo-arthrosis. Annals of the rheumatic diseases. 1957;16(4):494-502. Epub 1957/12/01.

15. Davis TJ, Shelbourne KD, Klootwyk TE. Correlation of the intercondylar notch width of the femur to the width of the anterior and posterior cruciate ligaments. Knee surgery, sports traumatology, arthroscopy : official journal of the ESSKA. 1999;7(4):209-14. Epub 1999/08/26. 
16. Dienst M, Schneider G, Altmeyer K, Voelkering K, Georg T, Kramann B, et al. Correlation of intercondylar notch cross sections to the $A C L$ size: a high resolution MR tomographic in vivo analysis. Arch Orthop Trauma Surg. 2007;127(4):253-60. Epub 2006/06/30.

17. Triantafyllidi E, Paschos NK, Goussia A, Barkoula NM, Exarchos DA, Matikas TE, et al. The shape and the thickness of the anterior cruciate ligament along its length in relation to the posterior cruciate ligament: a cadaveric study. Arthroscopy : the journal of arthroscopic \& related surgery : official publication of the Arthroscopy Association of North America and the International Arthroscopy Association. 2013;29(12):1963-73. Epub 2013/11/30.

18. Al-Saeed O, Brown M, Athyal R, Sheikh M. Association of femoral intercondylar notch morphology, width index and the risk of anterior cruciate ligament injury. Knee surgery, sports traumatology, arthroscopy : official journal of the ESSKA. 2013;21(3):678-82. Epub 2012/05/04. 19. Everhart JS, Flanigan DC, Simon RA, Chaudhari AM. Association of noncontact anterior cruciate ligament injury with presence and thickness of a bony ridge on the anteromedial aspect of the femoral intercondylar notch. The American journal of sports medicine. 2010;38(8):1667-73. Epub 2010/05/22.

20. Fung $D T$, Zhang LQ. Modeling of $A C L$ impingement against the intercondylar notch. Clin Biomech (Bristol, Avon). 2003;18(10):933-41. Epub 2003/10/29.

21. Hernigou P, Garabedian JM. Intercondylar notch width and the risk for anterior cruciate ligament rupture in the osteoarthritic knee: evaluation by plain radiography and CT scan. Knee. 2002;9(4):313-6. Epub 2002/11/09.

22. LaPrade RF, Burnett QM, 2nd. Femoral intercondylar notch stenosis and correlation to anterior cruciate ligament injuries. A prospective study. Am J Sports Med. 1994;22(2):198-202; discussion 3. Epub 1994/03/01.

23. Lombardo S, Sethi PM, Starkey C. Intercondylar notch stenosis is not a risk factor for anterior cruciate ligament tears in professional male basketball players: an 11-year prospective study. The American journal of sports medicine. 2005;33(1):29-34. Epub 2004/12/22.

24. Lund-Hanssen H, Gannon J, Engebretsen L, Holen KJ, Anda S, Vatten L. Intercondylar notch width and the risk for anterior cruciate ligament rupture. A case-control study in 46 female handball players. Acta Orthop Scand. 1994;65(5):529-32. Epub 1994/10/01.

25. Motohashi M. Profile of bilateral anterior cruciate ligament injuries: a retrospective follow-up study. J Orthop Surg (Hong Kong). 2004;12(2):210-5. Epub 2004/12/29.

26. Shelbourne KD, Facibene WA, Hunt JJ. Radiographic and intraoperative intercondylar notch width measurements in men and women with unilateral and bilateral anterior cruciate ligament tears. Knee surgery, sports traumatology, arthroscopy : official journal of the ESSKA. 1997;5(4):22933. Epub 1997/01/01.

27. Souryal TO, Freeman TR. Intercondylar notch size and anterior cruciate ligament injuries in athletes. A prospective study. Am J Sports Med. 1993;21(4):535-9. Epub 1993/07/01.

28. Souryal TO, Moore HA, Evans JP. Bilaterality in anterior cruciate ligament injuries: associated intercondylar notch stenosis. The American journal of sports medicine. 1988;16(5):449-54. Epub 1988/09/01.

29. Stijak L, Herzog RF, Schai P. Is there an influence of the tibial slope of the lateral condyle on the ACL lesion? A case-control study. Knee surgery, sports traumatology, arthroscopy : official journal of the ESSKA. 2008;16(2):112-7. Epub 2008/02/02.

30. Teitz CC, Lind BK, Sacks BM. Symmetry of the femoral notch width index. The American journal of sports medicine. 1997;25(5):687-90. Epub 1997/09/26.

31. van Diek FM, Wolf MR, Murawski CD, van Eck CF, Fu FH. Knee morphology and risk factors for developing an anterior cruciate ligament rupture: an MRI comparison between ACL-ruptured and non-injured knees. Knee surgery, sports traumatology, arthroscopy : official journal of the ESSKA. 2013. Epub 2013/07/09.

32. van Eck CF, Martins CA, Vyas SM, Celentano U, van Dijk CN, Fu FH. Femoral intercondylar notch shape and dimensions in ACL-injured patients. Knee surgery, sports traumatology, arthroscopy : official journal of the ESSKA. 2010;18(9):1257-62. Epub 2010/04/15. 
33. Vyas S, van Eck CF, Vyas N, Fu FH, Otsuka NY. Increased medial tibial slope in teenage pediatric population with open physes and anterior cruciate ligament injuries. Knee surgery, sports traumatology, arthroscopy : official journal of the ESSKA. 2011;19(3):372-7. Epub 2010/08/03. 